\title{
Application of Risk Factor Analysis Method in Bid Quotation
}

\author{
ZUO Ji-feng ${ }^{1}$, WANG Xue-chang ${ }^{2}$, LI Xue-fei ${ }^{1}$ \\ ${ }^{1}$ College of Science, School of Science Agricultural University of Hebei, Baoding 071001, China \\ ${ }^{2}$ Basic Course Department, Heibei College of Science and Technology, Baoding 071000, China
}

zjf79@sohu.com

Keywords: Risk analysis; Fuzzy mathematics; Bid quotation

\begin{abstract}
In this paper, a new method of weight determination and fuzzy mathematics is introduced, and the characteristics of risk factors of project bidding quotation are introduced, and a quantitative method for the risk analysis of bidding quotation is put forward.
\end{abstract}

\section{Introduction}

The risk factors affect the tender offer are mainly three types: [1] the bidders factors; [2]the engineering factors, such as design, construction and other factors; [3] the owners factors, namely, the owners credit and financial factors and so on . In particular, when bidders determine to tender ,they mainly think about ten risk factors which as follows: the management conditions, the conditions of the workers, the designers conditions, machinery and equipment conditions, project conditions, the same type project experience, the owners financial conditions, the contract conditions, the conditions of competition and future opportunities.

\section{The risk factors that affect the tender offer}

Bidding is a widely used international construction contracting methods,but due to the implementation of the system in our country is not long time, The lack of theory and practical experience of the loopholes have resulted in China's contractor bidding work in practice there have been many problems.

By introducing a new method to determine the weight and fuzzy mathematical method, combined with project risk factors, authors present a new method of tender offer quantitative risk analysis. Through a period of argument, I believe that the above-mentioned research results, and feasible, easy to accept, and in line with market rules, both in civil engineering and some specific engineering , it have a larger value of application.

\section{The method which analyze the risk factors of tender offer}

In this paper, the method which analyze the risk factor of tender offer is an expert evaluation method. Namely, before a new tender, firstly, determine the attribute weight $W$ of above ten factors, then use the ten factors to measure the project, according to the method of fuzzy mathematics divided each factors into five rank, give score for each rank as $C(1.0,0.8,0.6,0.4,0.2)$, according to various experts on the scoring of each factor to calculate the average score of each factor $C$, and then multiplied by $W$ and $C$, get the sum $\Sigma W C$, compared the sum to past similar projects, then determine to tender or not .If we determine to tender, deal with the risk based on the past similar project experience of tender offer.

Use the following method to determine the weight of risk factors:

Assumes the factors which affect the risk of tender offer are $\mathrm{A}_{1}, A_{2} \Lambda A_{n}(n=10)$, There are 10 experts judged

About the 10 factors, first to identify the most important factor and the most unimportant factor, according to Analytic Hierarchy Process (AHP) in the 1-9 scale and standard-setting between the 
two ratios.

Sort the 10 factors base on importance from small to large, the most unimportant factor as the base (assigned to 1) which will be compared with each factor.. Assignment according to importance (according to AHP method in the calibration and standards).

Divided the $n$-factors which evaluated by $m$ experts into $r$ blocks, respectively, denoted by $\mathrm{A}[1], \mathrm{A}[2], \ldots \mathrm{A}[r]$, Among ,each row in matrix $\mathrm{A}[k]$ represent the number of expert who think $A_{k}$ as the most unimportant factor, denoted by $m_{k}$. The column in the matrix represent the value of the $\mathrm{n}$-factors- $\mathrm{A}_{1}, A_{2} \Lambda A_{n}$, based on $A_{k}$. Concrete form as follows:

$A[k]=\left|\begin{array}{cccccccc}A_{1} & A_{2} & A_{k-1} & \Lambda & A_{k} & A_{k+1} & \cdots & A_{n} \\ A_{11}^{k} & A_{21}^{k} & A_{1, k-1}^{k} & K & 1 & A_{1, k+1}^{k} & K & A_{1 n}^{k} \\ A_{21}^{k} & A_{22}^{k} & A_{2, k-1}^{k} & K & 1 & A_{2, k+1}^{k} & K & A_{2 n}^{k} \\ A_{m 1}^{k} & A_{m 2}^{k} & A_{m, k-1}^{k} & K & 1 & A_{m, k+1}^{k} & K & A_{n n}^{k}\end{array}\right|$

Among, $\quad a_{i, k}^{k}=1,1 \leq a_{i, j}^{k} \leq 9$, and $\sum_{k=1}^{m} m_{k}=m\left(i=1,2, \Lambda m_{k}, j=1,2, \Lambda n\right)$.

For the block matrixA[k], due to various factors are assignment as $A_{k}$, a benchmark, we can averaged for each column in the matrix $\mathrm{A}[k]$.

$$
a_{j}^{k}=\sum_{i=1}^{m k} a_{i j}^{k} / m_{k}, j=1,2 \Lambda n,
$$

For all sub-block matrix make treatment as the above, we can get $\left(\mathrm{A}_{1}, A_{2} \Lambda A_{n}\right)$.For each sub-block matrixA[ $k](\mathrm{k}=1,2 \Lambda r)$; due to the number of rows is different,so the proportion in the number experts are also different, therefore need to consider the proportion of $m_{k}$ in $m$, say $\frac{m_{k}}{m}$ as the weight of $a_{j}^{k}$.

We can get the value of factor $A_{j}$. from above analysis.

$A_{j}=\sum_{k=1}^{r} a_{j}^{k^{*}} m_{k} / m, \quad \mathrm{j}=1,2 \Lambda \mathrm{n}$.

From the above analysis that can get $\mathrm{m}$ experts give the comprehensive assignment to the $\mathrm{m}$ factors. Derived by a combination of assignment $a_{j}$ in the minimum $a_{\min }$ and maximum $a_{\max }$ ordered by the corresponding subscript, respectively $m$ and $M$ that $a_{\min }=a_{m}, a_{M}=$ $a_{\max }$.

To $A[k]$ of all columns divided by the $a_{m}$,respectively corresponding to the column be:

When $m \mathrm{~m}$ is equal to $k$, that, $A[k]=A[k]$

When $m$ is not equal to $k$, that, $A[k]=\left(a_{i j}^{k} / a_{i m}^{k}\right) m * n$

$A_{m}, A_{M}$ in $\mathrm{A}[k]$ in the proportion of $p_{\min }^{k}$ and $p_{\max }^{k}$ as follows:

$p_{\text {min }}^{k}=\frac{m_{k}}{\sum_{i=1}^{m k} \sum_{j=1}^{n} \frac{a_{i j}}{a_{i m}^{k}}}$

$p_{\max }^{k}=\frac{\frac{\sum_{i=1}^{m k} a_{i M}}{a_{i m}^{k}}}{\sum_{i=1}^{m k} \sum_{j=1}^{n} \frac{a_{i j}}{a_{i m}^{k}}}$

$p_{\min }=\sum_{k=1}^{r} p_{\min }^{k^{*}} m_{k} / m$

$p_{\max }=\sum_{k=1}^{r} p_{\max }^{k^{*}} m_{k} / m$

the comprehensive assignment of $\mathrm{p}=p_{\max } / p_{\min }$

Solution of the above comparison matrix can be constructed:

When $a_{i} \geq a_{j}$, then, $a_{i j}=\left(a_{i}-a_{j}\right)(p-1) /\left(a_{\max }-a_{\text {min }}\right)+1$

When $a_{i}<a_{j}$, then, $a_{i j}=1 /\left[\left(a_{i}-a_{j}\right)(p-1) /\left(a_{\max }-a_{\text {min }}\right)+1\right]$

From this matrix can be derived to determine the weight of each factor.

\section{A project risk analysis}

According to the expert evaluation method to determine a project of the 10 risk factors, the 
weight shown in Table 1.

Table 1 expert evaluation method in the determination of the weight

\begin{tabular}{|c|c|c|c|}
\hline Tender considerations & Weight & Experts to score & WC \\
\hline Management conditions & 0.15 & 0.7 & 0.11 \\
\hline Working conditions & 0.10 & 0.9 & 0.09 \\
\hline Designers conditions & 0.10 & 0.9 & 0.09 \\
\hline Machinery and equipment condition & 0.05 & 0.5 & 0.03 \\
\hline Project Conditions & 0.05 & 0.6 & 0.03 \\
\hline Experience in similar projects & 0.15 & 0.8 & 0.12 \\
\hline Financial terms of the owners & 0.10 & 0.7 & 0.07 \\
\hline Conditions of Contract & 0.15 & 0.6 & 0.09 \\
\hline The conditions of competition & 0.10 & 0.5 & 0.05 \\
\hline Future opportunities & 0.05 & 0.3 & 0.02 \\
\hline
\end{tabular}

According to bidders on past experience, the $\Sigma W C$ more than 0.6 can be determined bidding. In this case,$\Sigma W C=0.7$, therefore decided to tender bidders, then the value according to $\Sigma W C$ similar projects from the past experience of the tender offer to determine the project's risk strategy to determine the final tender offer.

\section{Conclusion}

Project tender offer is a process which full of uncertainty, the uncertainty of tender offer affected by the project size, complexity and technical content and other factors. Bidders face enormous risks in the tender offer process. Bidders want to win, they must analyze the risk factors which affect the tender offer, and taking the right treatment strategy to reduce or avoid the risk. This paper presents a quantitative risk analysis can assist bidders assess the risk analysis of tender offer, and based on past experience, determine the tender offer of new projects.

\section{Acknowledgments}

This work is supported by the Project of Baoding science and Technology Bureau (No.13zs002).

\section{References}

[1] Y. Shao, N. Deng, A novel margin-based twin support vector machine with unity norm hyperplanes, Neural Computing and Applications DOI 10.1007/s00521-012-0894-5 (2012) $1-9$.

[2] Z. Qi, Y. Tian, S. Yong, Robust twin support vector machine for pattern classification, Pattern Recognition 46(1) (2012) 305-316.

[3] Y. H. Shao, N. Y. Deng, Z. M. Yang, Least squares recursive projection twin support vector machine for classification, Pattern Recognition 45 (6) (2012) 2299-2307.

[4] X.F. He, J. Lou, Z. He, Iterative methods for solving variational inclusions in Banach spaces, J. Comput. Appl. Math., 203 (2007) 80-86.

[5] J. W. Peng, On a new system of generalized mixed quasi-variational-like inclusions with 
[6] $(H, \eta)$-accretive operators in real q-uniformly Banach spaces, Nonlinear Anal.(2007) doi:10.1016/j.na.2006.11.054.

[7] Zadeh L.A. Fuzzy sets. Information and control [J],1965(8):338 353.

[8] LOWEN R. Mathematics and fuzziness. Fuzzy Sets and Systems, 1988:1 3.

[9] Xu Gaofeng. Effective Tendering of Public Infrastructure Projects in China. Successful Financing and Risk Management of Asian Project, 1997:27 31.

[10]Fameley. Competitive Bidding: A Review of Theory and Empirical Work. Journal of Economic Dynamic and Control, 1992:132 135. 